\title{
Exciton and negative trion dissociation by an external electric field in vertically coupled quantum dots
}

\author{
B. Szafran, ${ }^{1,2}$ T. Chwiej, ${ }^{1,2}$ F. M. Peeters, ${ }^{1}$ S. Bednarek, ${ }^{2}$ J. Adamowski, ${ }^{2}$ and B. Partoens ${ }^{1}$ \\ ${ }_{1}^{1}$ Departement Fysica, Universiteit Antwerpen (Campus Drie Eiken), Universiteitsplein 1, B-2610 Antwerpen, Belgium \\ ${ }^{2}$ Faculty of Physics and Applied Computer Science, AGH University of Science and Technology, al. Mickiewicza 30, \\ 30-059 Kraków, Poland
}

(Received 27 July 2004; revised manuscript received 15 November 2004; published 26 May 2005)

\begin{abstract}
We study the Stark effect for an exciton confined in a pair of vertically coupled quantum dots. A single-band approximation for the hole and a parabolic lateral confinement potential are adopted which allows for the separation of the lateral center-of-mass motion and consequently for an exact numerical solution of the Schrödinger equation. We show that for intermediate tunnel coupling the external electric field leads to the dissociation of the exciton via an avoided crossing of bright and dark exciton energy levels which results in an atypical form of the Stark shift. The electric-field-induced dissociation of the negative trion is studied using the approximation of frozen lateral degrees of freedom. It is shown that in a symmetric system of coupled dots the trion is more stable against dissociation than the exciton. For an asymmetric system of coupled dots the trion dissociation is accompanied by a positive curvature of the recombination energy line as a function of the electric field.
\end{abstract}

DOI: 10.1103/PhysRevB.71.205316

PACS number(s): 73.21.La, 71.35.Pq, 73.21.Fg

\section{INTRODUCTION}

Strained self-assembled InAs/GaAs quantum dots grown on subsequent layers stack spontaneously one above the other ${ }^{1,2}$ forming artificial molecules with spatially extended states due to the tunnel interdot coupling. The photoluminescence (PL) spectrum of the coupled dots consists of a number of lines which are blue or redshifted by the coupling 2,3 depending on the way the single-particle electron and hole wave functions contribute to the exciton states in question. ${ }^{4}$ Application of an electric field oriented along the growth direction offers the possibility of external control of the strength of the tunnel coupling. Recent experimental results ${ }^{5}$ on the Stark effect for vertically coupled pairs of nonidentical dots showed the effect of tunnel coupling through the appearance of avoided crossings between states localized in different dots. Previously, tunnel-coupling related Stark shift of the electroabsorption spectra has been observed in vertical stacks of several quantum dots. ${ }^{6}$

Stark effect on the exciton states in vertically coupled self-assembled quantum dots has previously been studied in Refs. 7 and 8. An anomaly in the ground-state Stark shift was found ${ }^{7}$ by the $\mathbf{k} \cdot \mathbf{p}$ method accounting for the strain effects and realistic shapes of the dots. This anomaly consists in deviation of the ground-state energy line from the usual quadratic dependence ${ }^{9}$ on the external field

$$
E(F)=E\left(F_{0}\right)-p\left(F-F_{0}\right)-\beta\left(F-F_{0}\right)^{2},
$$

where $F_{0}$ is the electric field for which the overlap of the electron and the hole wave functions is the largest and for which the recombination energy is maximal, $p$ is the dipole moment and $\beta>0$ - the polarizability. The shift calculated ${ }^{7}$ for coupled dots can only be approximated with two parabolas: one for $F<F_{0}$ and the other for $F>F_{0}$, amounting in a cusp at $F_{0}$. Although this deviation was attributed ${ }^{7}$ to the strain distribution it was shown that such a behavior can also be obtained in a single band model of coupled quantum disks neglecting the strain. ${ }^{8}$ Actually, as we discuss below analyzing the Stark shift of the first excited state, this deviation is due to a near degeneracy of the ground-state around $F_{0}$ resulting from the weakness of the hole tunnel coupling. In the present paper we report on another deviation of the Stark shift from quadratic form related to the exciton dissociation via a ground-state anticrossing of a bright state with both carriers in the same dot and a dark state with separated carriers.

Quantitative modeling ${ }^{10-19}$ of single quantum dots requires taking into account the valence band mixing, the gradient in the indium distribution, strain effects, and confinement geometry which are very different for quantum dots fabricated at various laboratories. In this paper we present a qualitative study of the effects of the external electric field on the interdot tunnel coupling visible in the Stark shifts of the bright energy levels, which should be universal for various types of coupled dots. In particular we focus on the effect of the electron-hole interaction which was neglected ${ }^{7}$ or treated in an approximate manner ${ }^{8}$ in previous work. For a single quantum dot the Coulomb interaction may have a small effect on the Stark shift since the interaction energy only weakly changes with the small displacement of the electron and hole wave functions inside a single dot. On the other hand, the role of the interaction for the Stark effect in coupled dots is essential since the effect of the external field on the exciton consists in breaking the electron-hole binding and segregation of carriers into different dots.

In the present work we use a simple model potential ${ }^{25}$ with a square quantum well for the vertical confinement and parabolic lateral confinement adopting the single band approximation for the hole. Due to the applied idealizations the model is exactly solvable. Our results fully account for the interparticle correlations due to the Coulomb interaction and cover also the excited states. 
A recent experiment ${ }^{5}$ on the Stark effect in a vertically coupled system of quantum dots was performed on a charge tunable structure, similar to the one used in studies of negatively charged excitons. ${ }^{20} \mathrm{~A}$ spectacular change in the spectrum was observed, ${ }^{5}$ when an electron was trapped in the dot closer to the electron reservoir. Namely, a sudden drop of the recombination energy and an unexplained positive curvature of the recombination line as a function of the electric field was observed. ${ }^{5}$ This observation motivated us to look at the Stark effect for the negatively charged trion. For the negative trion we apply the approximation that the lateral degrees of freedom are frozen. The validity of this approximation is first verified for the Stark shift of the exciton energy levels. In nanostructures the trion binding energies with respect to the dissociation into an exciton and a free electron are considerably increased. ${ }^{21}$ However, the trion binding energy is usually substantially smaller than the exciton binding energy. We report here that for a symmetric system of vertically coupled quantum dots the trion is more stable for dissociation by the external electric field than the exciton. The study of the dissociation mechanism shows, that for the pair of identical dots the trion is dissociated into a pair of electrons confined in one dot and a hole in the other. Only for the asymmetric system of coupled dots a dissociation into an exciton and a free electron is obtained as an intermediate step before the final separation of the hole from the two electrons. In this case, the trion is more easily dissociated than the exciton. The positive curvature of the recombination energy as a function of the electric field is obtained for the trion ionization process into an exciton and a free electron.

Previously, trions in vertically coupled dots were studied in the absence of the external field ${ }^{22}$ and neglecting tunnel coupling between the dots. ${ }^{23}$

This papers is organized as follows, the next section contains the description of the theoretical approach, the results are given in Sec. III, their discussion is presented in Sec. IV. Section V is devoted to the summary and conclusions.

\section{THEORY}

We assume a parabolic lateral confinement potential with equal electron and hole confinement energy $(\hbar \omega)$. Vertical confinement for the electron $\left[V_{e}\left(z_{e}\right)\right]$ and the hole $\left[V_{h}\left(z_{h}\right)\right]$ is taken as double well potentials of depth $V_{e}^{0}$ for the electron and $V_{h}^{0}$ for the hole and of width $w=6 \mathrm{~nm}$ separated by a barrier of thickness $b$. Isolated quantum dots may possess a built-in strain-induced electric field pushing the hole to the top of the dot as found in the photocurrent measurements of the Stark effect on buried quantum dots. ${ }^{24}$ However, in coupled quantum dots the built-in electric field has the opposite orientation. ${ }^{5}$ Therefore, this intrinsic electric field is neglected in the present calculations (in fact, such a build in electric field can also be interpreted as a shift of our applied field). For self-assembled quantum dots the assumption of harmonic lateral confinement is not valid, however, it should not essentially modify the susceptibility of the carriers to the electric field oriented vertically.

In the present model the Hamiltonian of the system can be written as

$$
\begin{aligned}
H= & -\frac{\hbar^{2}}{2 m_{e}} \nabla_{e}^{2}-\frac{\hbar^{2}}{2 m_{h}} \nabla_{h}^{2}+\frac{m_{e} \omega^{2}}{2} \rho_{e}^{2}+\frac{m_{h} \omega^{2}}{2} \rho_{h}^{2}+V_{e}\left(z_{e}\right)+V_{h}\left(z_{h}\right) \\
& -\frac{e^{2}}{4 \pi \epsilon \epsilon_{0} r_{e h}}+e \Phi\left(z_{e}\right)-e \Phi\left(z_{h}\right),
\end{aligned}
$$

where $\rho_{e}^{2}=x_{e}^{2}+y_{e}^{2},\left(x_{e}, y_{e}, z_{e}\right)$ and $\left(x_{h}, y_{h}, z_{h}\right)$ are the position vectors of electron and the hole, respectively. $r_{e h}$ is the electron hole distance, $m_{e}\left(m_{h}\right)$ is the electron (hole) effective band mass, $\epsilon$ is the dielectric constant, and $\Phi(z)$ is the potential of the external electric field taken as

$$
\Phi(z)=\left\{\begin{array}{ccc}
F z_{\max } & \text { for } & z_{\max } \leqslant z \\
F z & \text { for } & z_{\min }<z<z_{\max } \\
F z_{\min } & \text { for } & z \leqslant z_{\min }
\end{array}\right.
$$

where $F$ is the value of the electric field assumed to be uniform between $z_{\min }$ and $z_{\max }$ (which can be identified as the positions of the electrodes). In the calculations we leave a space of $10 \mathrm{~nm}$ between the dots and the points $z_{\min }$ and $z_{\max }$ beyond which the electric field is assumed to be zero.

The model of the coupled quantum dots used in this paper was previously applied ${ }^{25}$ to describe the exciton coupling between dots in the absence of an external electric field. The authors ${ }^{25}$ used the configuration interaction scheme to account for the lateral correlations between the electron and the hole. The configuration interaction approach for the electronhole systems is computationally much more challenging than for the electron systems due to its slow convergence. ${ }^{26}$ Therefore, in this paper we will make explicit use of the lateral separability of the center of mass. After introduction of the lateral relative $\boldsymbol{\rho}_{e h}=\left(x_{e}-x_{h}, y_{e}-y_{h}\right)$ and lateral centerof-mass $\boldsymbol{\rho}_{c m}=\left(m_{e} x_{e}+m_{h} x_{h}, m_{e} y_{e}+m_{h} y_{h}\right) / M$ coordinates, the Hamiltonian can be expresses as a sum of the lateral centerof-mass Hamiltonian $\left(H_{c m}\right)$ and the Hamiltonian for the relative lateral-and the single-particle vertical-motion $\left(H_{r v}\right)$, which are given by

$$
H_{c m}=-\frac{\hbar^{2}}{2 M} \nabla_{\rho_{c m}}^{2}+\frac{M \omega^{2}}{2} \rho_{c m}^{2}
$$

and

$$
\begin{aligned}
H_{r v}= & -\frac{\hbar^{2}}{2 \mu} \nabla_{\rho_{e h}}^{2}-\frac{\hbar^{2}}{2 m_{e}} \frac{\partial^{2}}{\partial z_{e}^{2}}-\frac{\hbar^{2}}{2 m_{h}} \frac{\partial^{2}}{\partial z_{h}^{2}}+\frac{\mu \omega^{2}}{2} \rho_{e h}^{2}+V_{e}\left(z_{e}\right) \\
& +V_{h}\left(z_{h}\right)-\frac{e^{2}}{4 \pi \epsilon \epsilon_{0} r_{e h}}+e \Phi\left(z_{e}\right)-e \Phi\left(z_{h}\right)
\end{aligned}
$$

with $M=m_{e}+m_{h}, \mu=m_{e} m_{h} /\left(m_{e}+m_{h}\right), \nabla_{\rho}^{2}$ stands for the Laplacian in the $x-y$ plane. The exciton wave function can be written as

$$
\Psi\left(\mathbf{r}_{\mathbf{e}}, \mathbf{r}_{\mathbf{h}}\right)=\chi\left(\boldsymbol{\rho}_{e h}, z_{e}, z_{h}\right) \psi_{c m}\left(\boldsymbol{\rho}_{c m}\right)
$$

where $\chi$ and $\psi_{c m}$ are the eigenfunctions of the $H_{r v}$ and the $H_{c m}$ Hamiltonians, respectively. Functions $\psi_{c m}$ are simply the eigenfunctions of a two-dimensional harmonic oscillator. 
The eigenstates of Hamiltonian (5) have definite $z$ component of total angular momentum and for $F=0$ also have definite parity with respect to a change of sign of the $z$ coordinates. ${ }^{4}$ The absorption/recombination probability for state $\mu$ is proportional to the integral

$$
\begin{aligned}
p_{\mu} & =\left|\int d^{6} \mathbf{r} \Psi_{\mu}\left(\mathbf{r}_{\mathbf{e}}, \mathbf{r}_{\mathbf{h}}\right) \delta^{3}\left(\mathbf{r}_{\mathbf{e}}-\mathbf{r}_{\mathbf{h}}\right)\right|^{2} \\
& =\left|\int d x_{e} d y_{e} \psi_{c m}\left(x_{e}, y_{e}\right) \int d z_{e} \chi_{\mu}\left(0, z_{e}, z_{e}\right)\right|^{2} .
\end{aligned}
$$

In the present paper, we consider only states whose symmetry does not prevent them to be bright, i.e., states in which both the relative $\chi$ and the center of mass $\psi_{c m}$ eigenstates possess zero angular momentum. In the following we show and discuss only results for states in which the center of mass is in the ground state. The spectrum with $s$-symmetry centerof-mass excitations is simply a replica of the spectrum corresponding to the ground state of the center of mass shifted by the energy $2 \hbar \omega$. The recombination probabilities for the states corresponding to zero angular momentum center-ofmass excitations are exactly equal to the corresponding states with the ground-state center of mass, since integrals of all the $s$ type wave functions of a two-dimensional harmonic oscillator are equal, which is due to a property of Laguerre polynomials. For potentials, in which the parity is a good quantum number, i.e., for identical quantum dots without an external field, we consider only states of even parity, the odd parity states being dark.

The eigenfunctions $\chi$ of Hamiltonian (5) are calculated on a three-dimensional finite-difference mesh with the imaginary time technique. ${ }^{27} \mathrm{We}$ use the material parameters for an $\mathrm{In}_{x} \mathrm{Ga}_{1-x}$ As quantum dot embedded in a GaAs matrix with a uniform concentration of indium in the quantum dot $x$ $=0.66 .{ }^{4}$ We take the following parameters for the alloyed quantum dot material $\epsilon=12.5, m_{e}=0.037 m_{0}, m_{h}=0.45 m_{0}$, where $m_{0}$ is the free electron mass, $V_{e}^{0}=-0.508 \mathrm{eV}, V_{h}^{0}$ $=-0.218 \mathrm{eV}$, and we take for the lateral confinement $\hbar \omega$ $=20 \mathrm{meV}$. We note, that in the limit of $\hbar \omega=0$ the present problem reduces to the Stark effect for an exciton in coupled quantum wells. ${ }^{28}$

For a particle of mass $m$ confined in a harmonic oscillator potential of energy $\hbar \omega_{0}$ the localization radius defined as the square root of the expectation value of $x^{2}+y^{2}$ is equal to $\sqrt{\hbar / m \omega_{0}}$. For the assumed center-of-mass separation the hole is therefore more strongly localized than the electron by a factor of $\sqrt{m_{h} / m_{e}}$. In InAs/GaAs quantum dots the hole confinement is stronger than the electron confinement which is due to the finite quantum well effect ${ }^{29}$ and the electron-hole interaction which localizes the heavy hole much more strongly than the light electron. In Fig. 7 we show that a change in the strength of the hole and electron lateral confinement does not influence the qualitative features of the spectra in an external electric field. It merely leads to shifts of the energy levels along the energy axis.

For the negative trion in quantum dots with a rectangularwell confinement the effect of a stronger hole localization leads to a larger electron-hole interaction energy than the electron-electron interaction energy. ${ }^{30}$ This produces a red- shift of the negative trion recombination line which increases with decreasing size of the dot and consequently leads to a decrease of the redshift due to the tunnel effect in coupled quantum dots. ${ }^{22}$ In two-dimensional quantum wells the experimentally observed ${ }^{31}$ positive and trion recombination energies for zero-magnetic field are nearly equal, although in strictly ${ }^{21}$ two-dimensional confinement significantly lower recombination energy for the positive trion was predicted. This effect is explained ${ }^{31-33}$ by stronger hole localization. Therefore, the adopted confinement potential takes into account the electron-hole interaction enhancement ${ }^{22,30-33}$ with respect to the electron-electron interaction.

\section{EXCITON IN VERTICALLY COUPLED DOTS}

\section{A. Stark effect}

For $F>0$ the electric field pushes the electron to the left and the hole to the right dot. The dependence of the energy spectrum on the external electric field for $b=2 \mathrm{~nm}$ is plotted in Fig. 1(a). At zero electric field the first excited state is of odd parity and corresponds to the excitation of the hole [see the inset to Fig. 1(a)-excitation energy is just $0.25 \mathrm{meV}$. The electric field breaks the parity symmetry of the system and the excited state becomes optically active [cf. the inset to Fig. 1(a)]. The dependence of the wave functions on the electric field is displayed in Fig. 2(a). In order to explain the field dependence of the spectrum we have plotted in Fig. 2(b) the probability densities integrated over the lateral degrees of freedom, which gives more accurate information about the localization of particles than the wave function on the axis [whose integral over $z_{e}$ and $z_{h}$ gives the recombination probability, cf. Eq. (7)]. In the ground state the hole becomes entirely localized in the right quantum dot for a relatively weak electric field [see the plots for $F=30 \mathrm{kV} / \mathrm{cm}$ in Figs. 2(a) and 2(b)]. The ground-state localization of the electron in the left dot appears at a much higher electric field, leading eventually to the extinction of the recombination intensity. In the excited part of the spectrum one observes two bright energy levels which tend to degeneracy at high electric field [cf. levels labeled by $b_{l}$ and $b_{r}$ in Figs. 1(a), 2(a), and 2(b)]. In these two energy levels the electron and the hole occupy the same quantum dot [it is more clearly visible in Fig. 2(b), for the wave function plots presented in Fig. 2(a) this tendency is apparent only at high electric field, cf. the plots for the third and fourth excited states for $F=90 \mathrm{kV} / \mathrm{cm}]$. In the bright energy levels marked by $b_{l}$ the carriers become localized in the left quantum dot which is favorable for the electrostatic energy of the electron and unfavorable for the electrostatic energy of the hole. In the higher bright energy level marked by $b_{r}$ the electron and the hole are localized in the right quantum dot, favorable for the hole and unfavorable for the electron. The $b_{l}$ level increases when the electric field is switched on. On the other hand the $b_{r}$ energy level decreases with field. This behavior is due to a reaction of the electron on the field which is delayed with respect to the reaction of the hole being more easily localized in one of the dots by the field [cf. Fig. 2(b)].

Figure 1(a) shows that the two bright energy levels exhibit avoided crossings and anticrossings with the dark energy lev- 

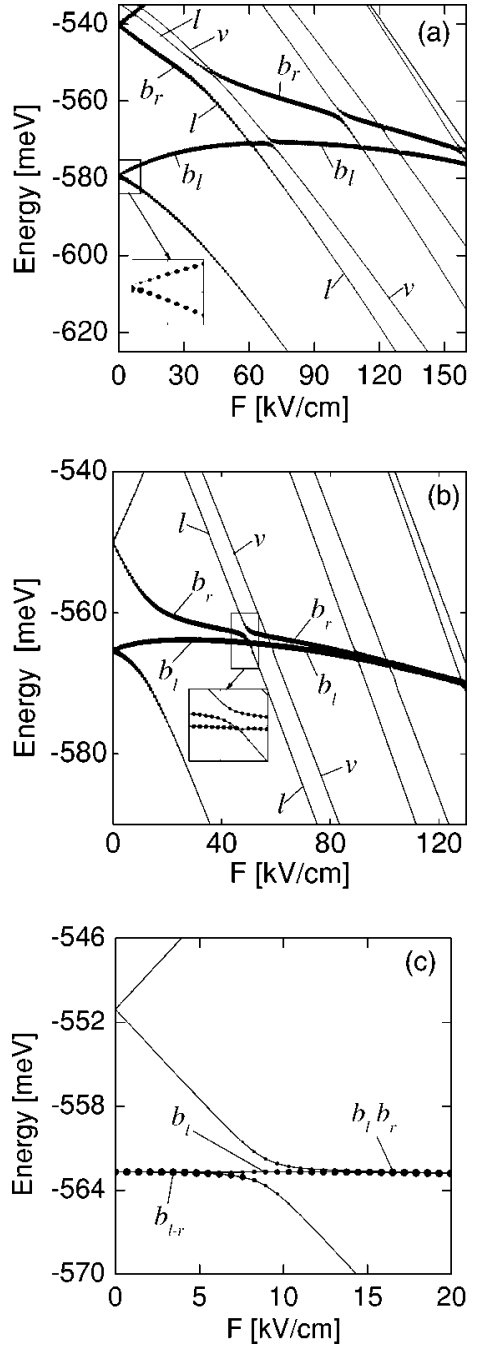

FIG. 1. Exciton energy spectrum as a function of external electric field $F$ for barrier thickness $b=2 \mathrm{~nm}$ (a), $b=4 \mathrm{~nm}$ (b), $b$ $=7 \mathrm{~nm}(\mathrm{c})$. The area of the dots is proportional to the recombination probability. The insets in (a) and (b) show zooms of regions marked by rectangles.

els for which the carriers are separated by the electric field in the same way as in the ground state. The lowest excited dark energy level [marked by $l$ in Figs. 1(a), 2(a), and 2(b)] corresponds to a lateral excitation. In the second excited dark energy level [marked by $v$ in Figs. 1(a), 2(a), and 2(b)] the hole in the right quantum dot is in a state excited in the vertical direction. For $b=2 \mathrm{~nm}$ the first anticrossing in the low-energy spectrum appears between the bright $b_{r}$ and the dark $l$ energy levels around $F=40 \mathrm{kV} / \mathrm{cm}$ at about $-555 \mathrm{meV}$. This anticrossing is wide and is due to the electron tunnel coupling of the left and right dots (the hole is entirely localized in the right quantum dot in both states). The dark energy level $l$ goes below the lower bright energy level $b_{l}$ via a crossing. A crossing instead of anticrossing is observed here because in the $b_{l}$ energy level the hole is in the other (left) dot. The dark state $v$ with a hole excitation crosses the $b_{r}$ level and goes below the $b_{l}$ level in a very narrow anticrossing.

For weaker tunnel coupling, i.e., for $b=4 \mathrm{~nm}$ [cf. Fig. 1(b)] the two bright energy levels become degenerate already at about $F=90 \mathrm{kV} / \mathrm{cm}$. All the avoided crossings become narrower with respect to the stronger tunnel coupling case of Fig. 1(a). The most pronounced anticrossing is the one between the $b_{r}$ and $l$ energy levels, like for $b=2 \mathrm{~nm}$ [cf. Fig. 1(a)]. The curvature of the degenerate bright energy levels at high electric fields results from the electric-field-induced deformation of the electron and hole wave functions within each of the quantum dots.

The most interesting spectrum is obtained for larger barrier thickness. Figure 1(c) displays the electric-field dependence of the exciton energy spectrum for $b=7 \mathrm{~nm}$. For $F=0$ the twofold degenerate ground state corresponds to both carriers in the same quantum dot [cf. Fig. 2(b)], while in the nearly degenerate excited state the carriers occupy different quantum dots. The degenerate ground state energy is not affected by the electric field, since the electrostatic energy gained by the electron is lost by the hole and vice versa. The electric-field dependence of both the split excited energy levels, which correspond to spatially separated charge carriers, is strictly linear. This energy level anticrosses the $b_{r}$ bright energy level around $F=9 \mathrm{kV} / \mathrm{cm}$. After the avoided crossing the state with carriers separated by the external electric field becomes the ground state. The bright state $b_{l}$ is not involved in the anticrossing and its energy is independent of $F$. For larger $b$ the discussed anticrossing becomes narrow and barely visible.

Figure 2(c) for $F=5 \mathrm{kV} / \mathrm{cm}$ shows that in the groundstate the charge of the hole is considerably shifted to the right dot and that a part of the electron charge is also transferred to the right dot. In order to present the movement of the carriers between the dots in more detail we plotted in Fig. 3 the charge accumulated in the left dot as a function of the electric field for different barrier thicknesses. We see that the dependence of the hole charge on the external field is monotonous. On the other hand the electron initially follows the movement of the hole to the right dot. For $b=10 \mathrm{~nm}$ the electron charge transferred to the right dot is exactly equal to the hole charge for $F$ smaller than $6 \mathrm{kV} / \mathrm{cm}$. Up to this field both quantum dots remain neutral and the dipole moment (see inset to Fig. 3) is zero. When both particles become completely localized in different dots the dipole moment reaches $e(b+w)$.

\section{B. Nonidentical quantum dots}

The confinement potential of vertically stacked dots usually exhibits asymmetry, which even for identical dots can be induced by the strain effects. ${ }^{7}$ Let us consider the effect of the asymmetry of the confinement potential on the exciton spectrum. It was established ${ }^{7}$ that for stacked strained truncated pyramids the ground state of the hole is completely localized in one of the dots, while the electron (noninteracting ${ }^{7}$ with the hole) still forms bonding and antibonding states.

Here, we simulate this type of localization assuming unequal depths of the quantum wells for the hole. The effect of the electric field on the spectrum of asymmetric coupled dots for $b=6 \mathrm{~nm}$ is presented in Fig. 4(a) for the right dot deeper by $3 \mathrm{meV}$ for the hole. Two bright energy levels around 
(a)

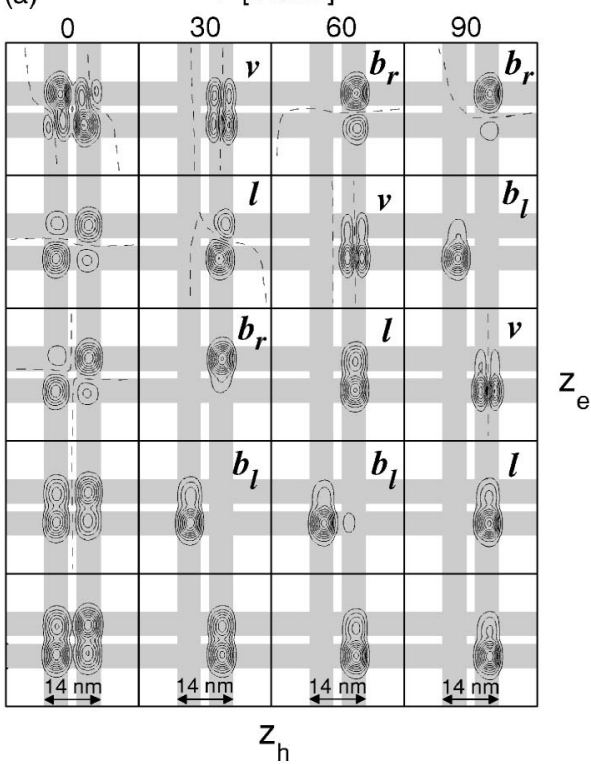

(b)

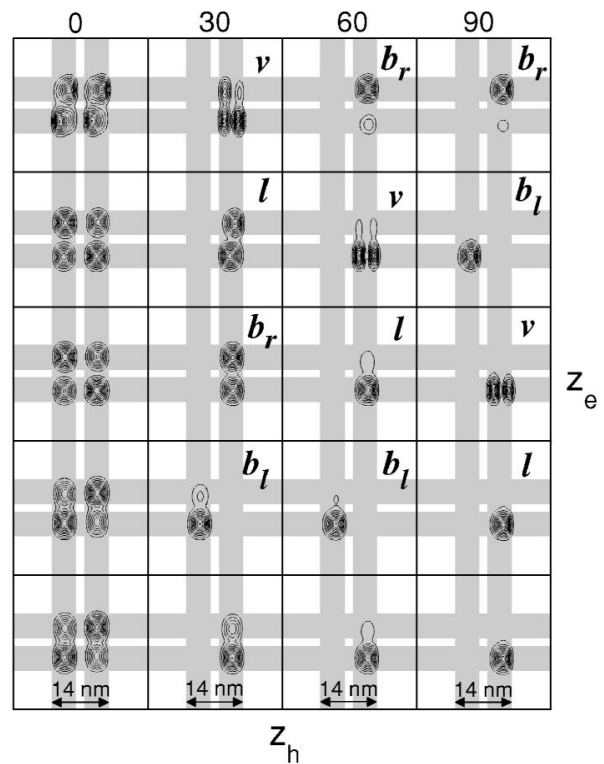

(c) $\quad \mathrm{F}[\mathrm{kV} / \mathrm{cm}]$

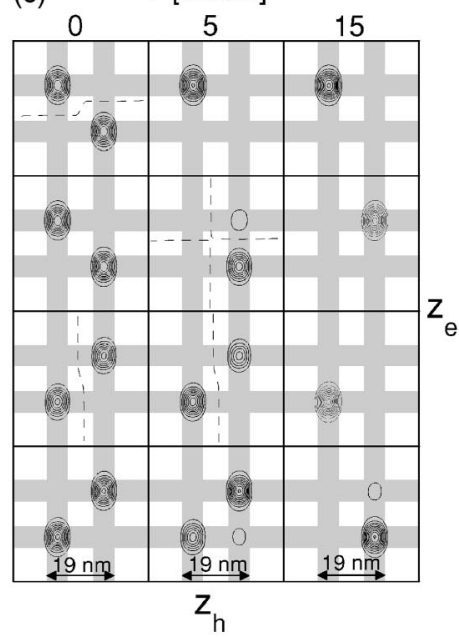

FIG. 2. (a), (c) contour plots of wave functions at the axis $\rho=0$ of the system and (b) probability density integrated over the lateral degrees of freedom $\int_{0}^{\infty} d \rho \rho\left|\chi\left(\rho, z_{e}, z_{h}\right)\right|^{2}$ for different values of the electric field for barrier thickness $b=2 \mathrm{~nm}(\mathrm{a})$, (b) and $b=7 \mathrm{~nm}$ (c). Lower plots correspond to lower energies. Shaded area show the quantum wells for the electron and for the hole. Dashed line shows the nodal surface of the wave function.

-563 and $-560 \mathrm{meV}$ are obtained. In the lower (upper) of energy levels both the carriers are localized in the deeper (shallower) of the dots. The lowest dark energy level decreasing linearly in energy with $F$ has the hole localized in the right dot [cf. Fig. 5(a)] so it crosses the higher bright energy level with both the carriers in the left dot. The interchange of the energy order of this dark state with the lower bright energy level appears via an avoided crossing, since in both these states the hole is localized in the right (deeper) dot [cf. Fig. 5(a)]. For $F<0$ the hole in the lowest energy dark state is localized in the shallower of the dots. For this reason the corresponding energy level anticrosses the higher bright energy level and crosses the lower one.

Let us now suppose that the left dot is shallower for the electron (by $3 \mathrm{meV}$ ) and that the hole confinement is symmetric. Figure 4(b) shows the spectrum for this case. Surprisingly the spectrum for the electron confinement asymmetry

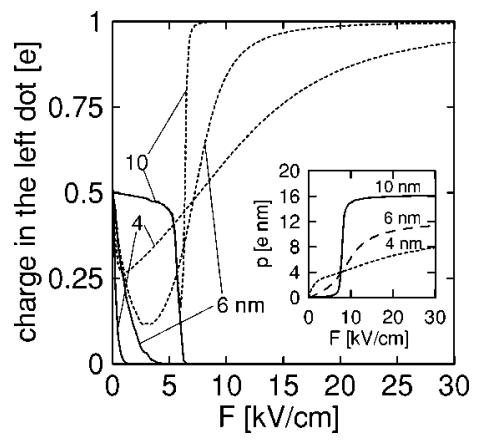

FIG. 3. Electron (dotted line) and hole (solid line) charge accumulated in the left quantum dot as function of the electric field for different barrier thicknesses. Inset shows the dipole moment as function of the field. is just shifted by $+2.5 \mathrm{kV} / \mathrm{cm}$ with respect to the spectrum for the hole confinement asymmetry [cf. Fig. 4(a)]. In the lower (upper) of the bright energy levels the electron stays in the deeper (shallower) of the dots and the Coulomb interaction binds the hole in the same dot [cf. Fig. 5(b)]. The crossing/anticrossing mechanism is the same as for the hole confinement asymmetry.

For smaller barrier thickness the anticrossings of the dark and bright energy levels become wider and as a consequence the region near $F=0$ in which the two lowest energy levels are nearly independent of $F$ is narrower. The spectra for the hole asymmetry for $b=4.5$ and $3 \mathrm{~nm}$ are displayed in Figs. 6(a) and 6(b), respectively. The two parallel energy levels near $F=0$ observed for weak tunnel coupling in Fig. 4 are now (see Fig. 6) converted into a crossing at a small negative $F$. This feature results in the cusp of the ground-state energy reported previously ${ }^{7}$ for a thin $(1.8 \mathrm{~nm})$ interdot barrier. For the electron asymmetry the spectra are still shifted to higher values of the field by about $2.5 \mathrm{kV} / \mathrm{cm}$ with respect to the hole asymmetry, like in the weak coupling case of Fig. 4. The crossing of the bright energy levels still appears at $F$ $<0$. The reason of this similarity is that in the ground state at $F=0$ the dipole moment induced by the electron and hole asymmetry is the same in sign and not very different in size. For small $b$ the electron charge is smeared over both dots. If the right dot is deeper for the electron it binds a larger part of its charge. Consequently, the entire charge of the hole is pulled to the right dot. On the other hand, for the hole confinement asymmetry the dot which is deeper for the hole localizes its charge completely even for small $b$ since the hole tunnel coupling is negligible. The localization of the hole in the right dot results in a larger localization of the electron in the right dot. In this way the asymmetry of the confinement for one particle is translated into an asymmetry 

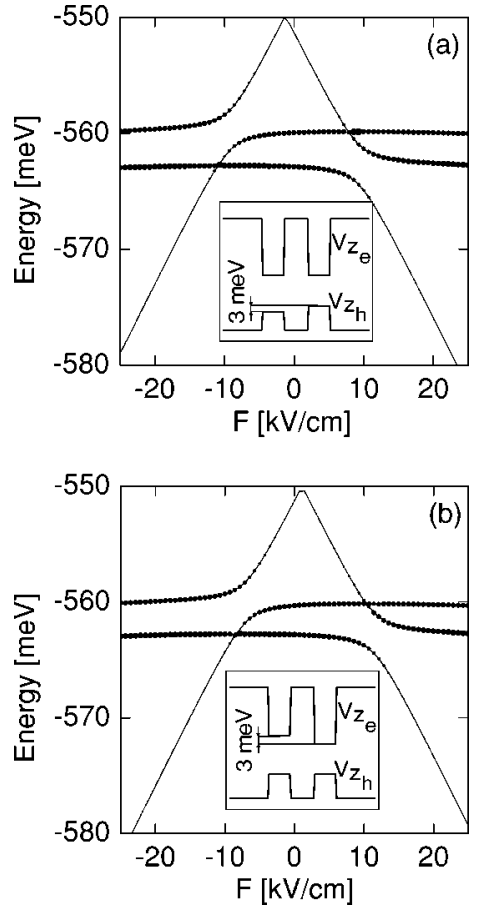

FIG. 4. Stark effect for the asymmetric system of quantum dots at $b=6 \mathrm{~nm}$. In (a) the electron confinement is symmetric and the left dot for the hole is shallower by $3 \mathrm{meV}$. In (b) the hole confinement is symmetric and the left dot for the electron is shallower by $3 \mathrm{meV}$. The insets in (a) and (b) show a schematic drawing of the vertical confinement for $F=0$.

of the potential felt by the other particle via the Coulomb interaction. Although it is possible experimentally to determine which of the dots is deeper by looking at the electricfield dependence of the bright energy levels one cannot deduce from the $F$ dependence of the exciton energy levels alone which of the carriers is responsible for the asymmetry.

\section{Frozen lateral degrees of freedom}

The exact separability of the center of mass used in the previous calculations was possible because of the assumption of identical lateral confinement energies for the electron and the hole. When the center of mass is not separable ${ }^{26}$ the exact calculations become much more complex. However, as long as the interest of calculations relies in a qualitative description of the influence of the electric field applied in the growth direction the actual form of the lateral confinement is not essential. In this case one may try to integrate out the lateral degrees of freedom. ${ }^{34,35}$ Such an adiabatic approximation is valid for strong lateral confinement, as in the case of selfassembled quantum dots. Thus we assume that the electron and hole lateral wave functions can be identified with the ground-state of the harmonic oscillator. This assumption allows us to integrate ${ }^{35}$ over the lateral degrees of freedom and arrive at the effective Hamiltonian for the vertical motion
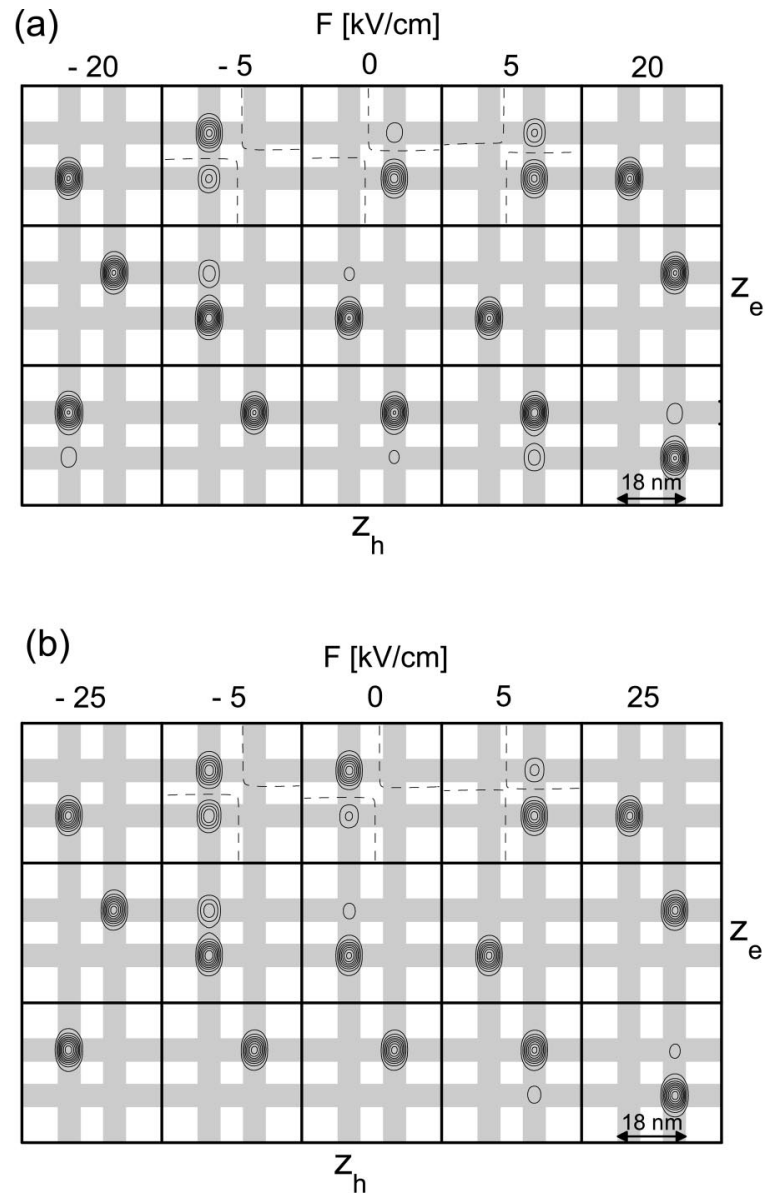

FIG. 5. (a) Contour plots of the wave functions at the axis $\rho$ $=0$ corresponding to the energy levels shown in Fig. 4(a). (b) similar as for Fig. 4(b). Higher plots correspond to higher energies.

$$
\begin{aligned}
H_{\text {eff }}= & -\frac{\hbar^{2}}{2 m_{e}} \frac{\partial^{2}}{\partial z_{e}^{2}}-\frac{\hbar^{2}}{2 m_{h}} \frac{\partial^{2}}{\partial z_{h}^{2}}+V_{e}\left(z_{e}\right)+V_{h}\left(z_{h}\right) \\
& -V_{\text {eff }}\left(\left|z_{e}-z_{h}\right|\right)+e \Phi\left(z_{e}\right)-e \Phi\left(z_{h}\right)+2 \hbar \omega,
\end{aligned}
$$

with $V_{\text {eff }}(z)$ the effective potential ${ }^{35}$ of one-dimensional interaction given by

$$
V_{\text {eff }}(z)=\frac{e^{2}}{4 \pi^{1 / 2} \epsilon \epsilon_{0} l} \operatorname{erfcx}(|z| / l),
$$

with $l=\sqrt{\hbar\left(1 / m_{e}+1 / m_{h}\right) / \omega}$. The solution to the eigenequation of the effective Hamiltonian (8) describes the effects appearing in the growth direction at the expense of a simplified picture of the lateral motion.

Figure 4 shows the comparison of the exact results (solid lines) obtained with the separated center-of-mass and approximate results calculated for frozen lateral degrees of freedom (dashed lines) for identical quantum dots separated by a barrier of thickness $b=4 \mathrm{~nm}$, as considered above in Fig. 4(b). The approximate method reproduces the correct qualitative shape of the energy lines. Also the recombination probability dependence on the electric field does not significantly differ. However, the approximation of the frozen lateral state eliminates the lateral excitations. The avoided 

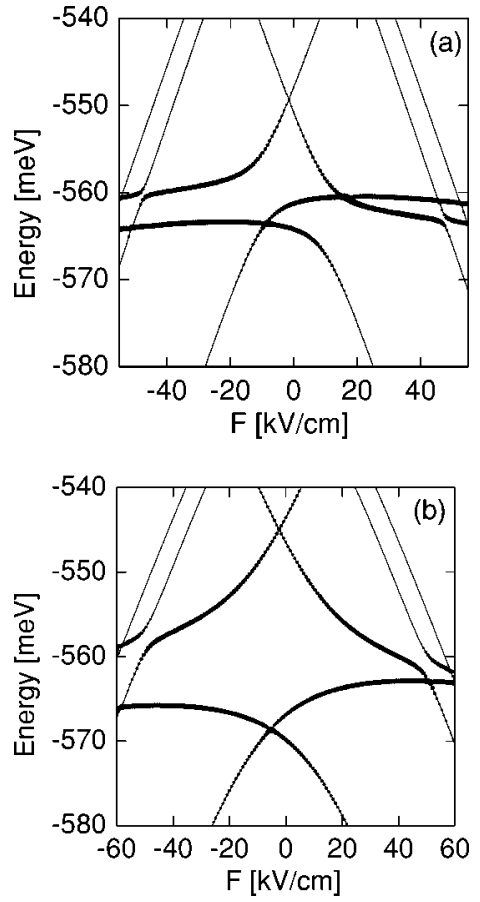

FIG. 6. Stark effect for the asymmetric hole confinement of Fig. 4(a) for $b=4.5 \mathrm{~nm}$ (a) and $b=3 \mathrm{~nm}$ (b). The area of the dots shows the recombination probability.

crossings of the bright energy levels with the dark energy levels with lateral excitations are therefore overlooked in the present approximation [cf. avoided crossing at $F$ $=50 \mathrm{kV} / \mathrm{cm}$ missing for lines marked with dashed lines]. The accuracy of the approximate method is better for dark states with separated charge carriers than for the bright energy levels for which the electrons and hole wave function overlap. ${ }^{35}$ The discussed approximation can be applied to evaluate the qualitative dependence of the bright energy levels on the external field when lateral excitations are absent. In the following section we will use this approach to study the effect of the external field on the negatively charged trion in coupled dots.

The dotted lines in Fig. 7 show the results of frozendegree-of-freedom calculations performed for the electron confinement unchanged but weakened hole confinement for which the lateral confinement radii of the electron and the hole are equal. For weakened hole confinement the electronhole interaction energy is smaller, which leads to a blueshift of the energy levels for $F=0$ with respect to the equal confinement energies case (dashed lines in Fig. 7). The interaction energy of the dissociated electron-hole pair is less strongly affected by the change of the hole localization strengths. Figure 7 shows that the electric-field dependence on the electric field is essentially not altered by the strength of the hole localization, which justifies a posteriori the assumption of the adopted center-of-mass separability.

\section{STARK EFFECT FOR NEGATIVE TRION}

We consider the effect of the electric field on the ground state of a negatively charged trion in which the electron sub-

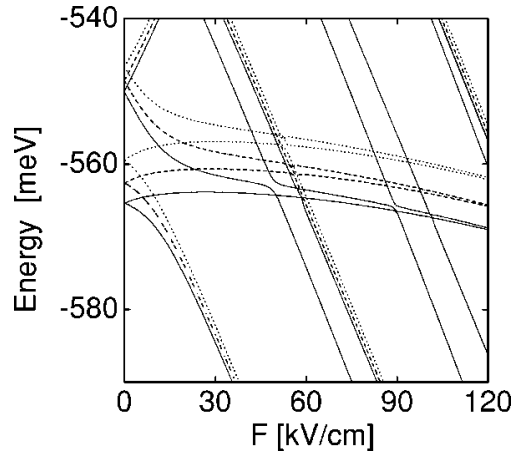

FIG. 7. The exact results solid lines and the results with the frozen degree of freedom for equal hole and electron confinement energies (dashed lines) and with equal hole and electron confinement lengths (dotted line) for the parameters of Fig. 4(b).

system is in the singlet state. The approximation of the frozen lateral wave functions will be used with the quasione-dimensional ${ }^{35}$ electron-electron $\left(V_{\mathrm{eff}}^{e e}\right)$ and electronhole interaction potential [Eq. (9)]. Electron-electron $V_{\mathrm{eff}}^{e e}$ potential is obtained for $m_{h}$ replaced by $m_{e}$ in formula (9). The Hamiltonian for the trion reads

$$
\begin{aligned}
H_{\mathrm{eff}}^{X-}= & -\frac{\hbar^{2}}{2 m_{e}}\left(\frac{\partial^{2}}{\partial z_{e 1}^{2}}+\frac{\partial^{2}}{\partial z_{e 2}^{2}}\right)-\frac{\hbar^{2}}{2 m_{h}} \frac{\partial^{2}}{\partial z_{h}^{2}}+V_{e}\left(z_{e 1}\right)+V_{e}\left(z_{e 2}\right) \\
& +V_{h}\left(z_{h}\right)-V_{\mathrm{eff}}\left(\left|z_{e 1}-z_{h}\right|\right)-V_{\mathrm{eff}}\left(\left|z_{e 2}-z_{h}\right|\right) \\
& +V_{\mathrm{eff}}^{e e}\left(\left|z_{e 2}-z_{e 1}\right|\right)+e \Phi\left(z_{e 1}\right)+e \Phi\left(z_{e 2}\right)-e \Phi\left(z_{h}\right)+3 \hbar \omega,
\end{aligned}
$$

where $z_{e 1}$ and $z_{e 2}$ are coordinates of the first and second electron, respectively.

Figure 8(a) shows the difference of the trion ground state energy and the ground state energy of a single electron as function of the electric field for different values of the barrier thickness and identical pair of quantum dots of width $6 \mathrm{~nm}$. The energy difference presented in Fig. 8(a) can be identified $^{22}$ with the energy of the photon released when the hole recombines with one of the electrons (calculated with respect to the GaAs energy gap similarly as for the exciton). For comparison the exciton ground state energy calculated with the same approximation of the frozen lateral states is also shown by the dashed lines. In the absence of the electric field the recombination line of the negative trion has a lower energy than the exciton recombination energy [cf. the inset to Fig. 8(a)]. We found that the redshift of the trion line is smaller for smaller barrier thickness. This behavior as obtained by neglecting the lateral correlations is in perfect qualitative agreement with extensive variational calculations accounting for both vertical and lateral correlations in a nearly exact way. ${ }^{22}$ Inset to Fig. 8(a) shows that for high $F$ the energy difference of the trion and exiton energy lines is an increasing function of $b$. This is due to the fact that the interaction energy between the electrons confined in the same dot is larger than the Coulomb interaction between the hole and electron separated by the barrier.

For larger barrier thickness [cf. plots for $b=6$ and $8 \mathrm{~nm}$ in Fig. 8(a)] the recombination line of the trion is independent 

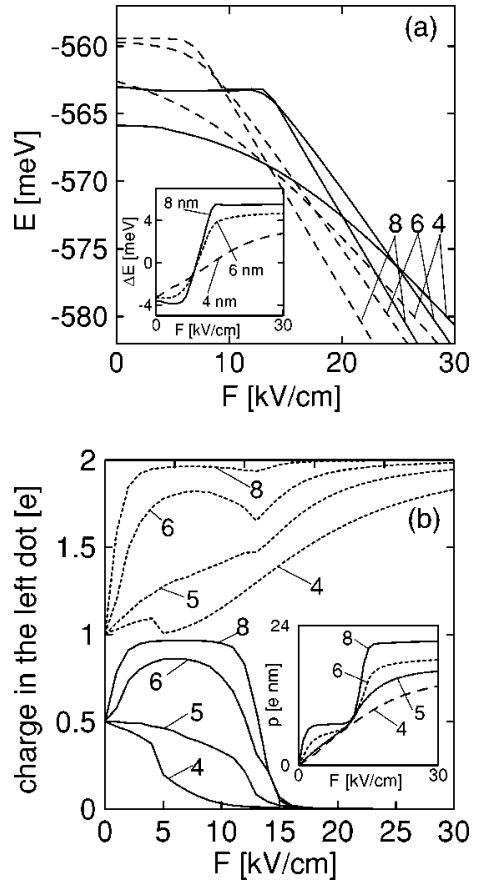

FIG. 8. (a) Difference of the ground-state trion energy and the electron ground state (trion recombination energy with respect to GaAs energy gap-solid lines) and the exciton ground-state energy (exciton recombination energy-dashed lines). The curves are labeled by the barrier thickness $b$ in nanometers. Inset shows the difference of the exciton and trion energy lines. (b) Electron (dotted lines) and hole (solid lines) charge on the left side of the origin as function of the electric field. Inset shows the dipole moment.

of the electric field for $F$ lower than about $13 \mathrm{kV} / \mathrm{cm}$. The flat part of the plots corresponds to both the electrons and the hole staying in the same quantum dot (as discussed above for exciton). We can see that the ground state of the trion is more resistant to the dissociation by the electric field than the exciton ground state. The exciton energy decreases faster than the trion recombination line, which results in the reversal of the order of the lines at $F=14 \mathrm{kV} / \mathrm{cm}$ for $b=4 \mathrm{~nm}$ and $F=10 \mathrm{kV} / \mathrm{cm}$ for $b=6$ and $8 \mathrm{~nm}$. For large values of $F$ for which the hole and the electron charges in both the exciton and the trion ground states are completely separated, the trion and exciton energy lines for each $b$ run parallel to each other.

To explain the large stability of the trion ground state in the symmetric coupled dots against dissociation by the electric field we plotted in Fig. 8(b) the hole and the electron charge accumulated in the left dot as a function of the electric field for different barrier thicknesses. For large $b$ the distribution of the electron and the hole charges between the dots before the dissociation of the trion is qualitatively different than in the exciton case (cf. Fig. 3). For $F=0$ the hole (electron) charge in the right dot is 0.5 (1) due to the symmetry of the system. For large barrier thickness $(b=8 \mathrm{~nm})$ the electrons become localized in the left dot already under the influence of a weak electric field. The hole initially follows the electrons into the left dot (cf. the local maximum of the solid line for $b=8 \mathrm{~nm}$ ). We remind the reader that for the exciton an opposite behavior was observed (cf. Fig. 3): the

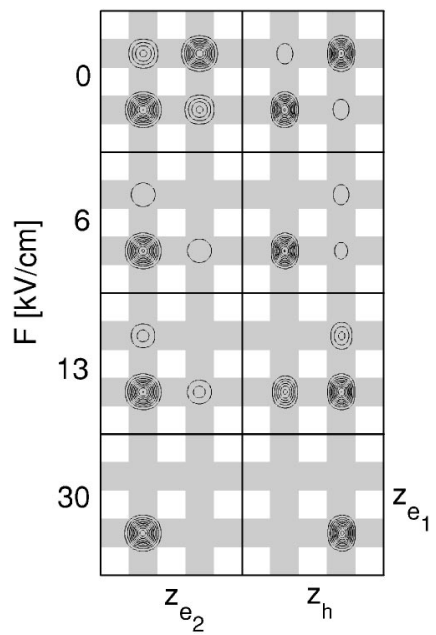

FIG. 9. Probability density integrated over the vertical coordinate of one of the electrons (right panel) and the vertical coordinate of the hole (left panel) for $b=6 \mathrm{~nm}$ and different values of the electric field $F$. The shaded areas show the positions of the quantum dots.

electron initially followed the hole for weak electric fields. The trion becomes dissociated around $13 \mathrm{kV} / \mathrm{cm}$, when the field moves the hole from the left to the right dot. The reaction of the carriers on the electric field is the most complex for $b=6 \mathrm{~nm}$ [cf. Fig. 8(b)]. We have illustrated this in Fig. 9 by additional plots of the probability densities integrated over the vertical coordinate of one the three particles. For zero electric field there is a nonzero probability of finding the electrons in different dots (cf. the left plot for $F=0$ in Fig. 9), and the probability of finding an electron in a different quantum dot than the hole (cf. the right plot for $F=0$ in Fig. 9) is much smaller. For $b>8 \mathrm{~nm}$ all the three particles are found in the same dot. The leakage of particles to the other dot seen in Fig. 9 is a result of the electron tunnel coupling which is already nonzero for $b=6 \mathrm{~nm}$. In contrast to the case of $b$ $=8 \mathrm{~nm}$, for $b=6 \mathrm{~nm}$ a part of the electron charge stays in the right dot when the field is switched on (cf. the left plot for $F=6 \mathrm{kV} / \mathrm{cm}$ in Fig. 9). When the hole is transferred to the right dot (cf. the plots for $F=13 \mathrm{kV} / \mathrm{cm}$, part of the electron charge follows it, which results in a local minimum of the electron charge accumulated in the left dot for $F$ around $13 \mathrm{kV} / \mathrm{cm}$ [cf. Fig. 8(b) for $b=6 \mathrm{~nm}$ ]. For larger $F$ the particles become separated. For stronger tunnel coupling between the dots, i.e., for $b=5$ and $4 \mathrm{~nm}$ the hole charge accumulated in the left dot depends on the external field monotonically [cf. Fig. 8(b)], and a part of the electron charge attempts to follow the hole when it leaves the left dot. Therefore, for small $b$ the mechanism of the trion resistance to dissociation becomes similar to the one observed for the exciton (cf. Fig. 3). The present results show that for symmetric quantum dots the trion becomes dissociated into a pair of electrons in one dot and the hole in the other without the intermediate step consisting of an exciton confined in the right dot and an electron in the left dot. This mechanism is more clearly pronounced for larger $b$. The Coulomb interaction of the electrons with the hole stabilizing the complex against field-induced dissociation without the intermediate step is two times larger than for exciton. 

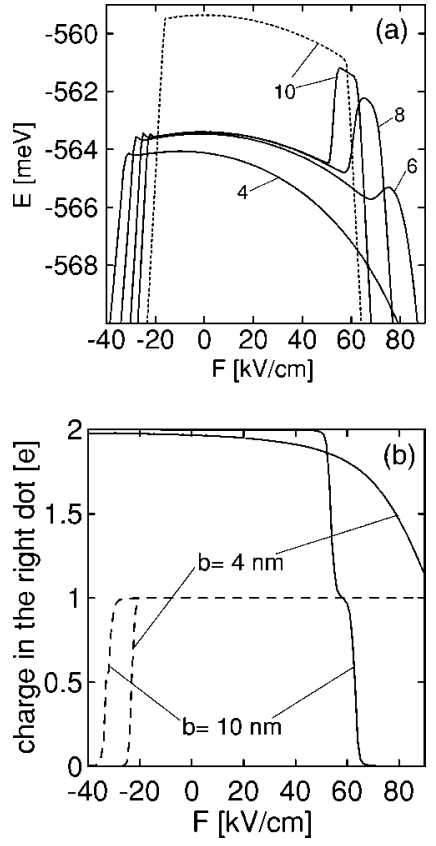

FIG. 10. (a) The trion recombination energies as functions of the electric field (solid lines) for a pair of coupled quantum dots. The curves are labeled by the barrier thickness in nanometers. Right dot has a width of $6 \mathrm{~nm}$ and the width of the left dot is $4 \mathrm{~nm}$. Dotted line shows the exciton recombination energy for $b=10 \mathrm{~nm}$. (b) The electron (solid lines) and the hole (dashed lines) charge accumulated in the right dot for $b=4$ and $10 \mathrm{~nm}$

Note that for the trion [cf. Fig. 8(b)] the barrier thickness has the opposite effect on the sensitivity of the electrons and the hole to the electric-field induced localization. For smaller $b$ the electric field is less effective in localizing the electrons in the left dot but more effective in localizing the hole in the right dot. The effect for the electrons is obviously due to the strong electron tunnel coupling. For smaller $b$ a smaller $F$ localizes the hole in the right dot because the energy of its interaction with electrons changes less drastically after the dissociation and the hole tunnel coupling is negligible.

The inset to Fig. 8(b) shows the electric dipole moment for the trion as a function of the electric field. For high $F$, when the particles are separated into different dots the dipole moment takes the value $3 e(w+b) / 2 .{ }^{36}$ Note, that for a thick barrier the dipole moment develops a plateau for the region of fields in which the hole accompanies the electrons to the right dot. For a thick barrier the recombination energy [cf. inset of Fig. 8(a)] starts to change only when the second plateau of the dipole moment is reached.

We found a qualitatively different dissociation mechanism of the trion in an asymmetric system of coupled dots. Suppose that the right quantum dot has a thickness of $6 \mathrm{~nm}$ (as anywhere else in the present paper) and that the left dot has a thickness of only $4 \mathrm{~nm}$. Figure 10(a) shows the trion recombination energies for different barrier thicknesses. The charge accumulated in the right (wider) dot is plotted in Fig. 10(b). For $F=0$ the three carriers stay in the right dot. For strongly coupled dots $(b=4 \mathrm{~nm})$ the electrons resist strongly to being removed to the thinnest dot. For $F=90 \mathrm{kV} / \mathrm{cm}$ less than one elementary charge is localized in the left dot. On the other hand the negative electric field removes abruptly the hole to the thinnest dot at $F<-25 \mathrm{kV} / \mathrm{cm}$. For thicker interdot barrier the trion recombination energy develops a local maximum for positive electric fields [see the plots for $b=6$, 8 , and $10 \mathrm{~nm}$ in Fig. 10(a)]. Let us analyze the origin of these maxima for the case of $b=10 \mathrm{~nm}$. For positive electric field up to $50 \mathrm{kV} / \mathrm{cm}$ both the electrons are confined in the right dot [cf. Fig. 10(b)]. Then between $F=50 \mathrm{kV} / \mathrm{cm}$ and $F=55 \mathrm{kV} / \mathrm{cm}$ one of the electrons is transferred to the right dot. In this electric field range the trion is dissociated into an exciton confined in the right dot and a spectator electron in the left quantum dot. The final state after the trion recombination, i.e., the ground state of a single electron, is localized in the left quantum dot for $F>50 \mathrm{kV} / \mathrm{cm}$, i.e., for the same value of the electric field which induces the transition of the first electron from the trion state to the left quantum dot. ${ }^{37}$ After the trion dissociation the recombination energy almost reaches the recombination energy of the exciton [cf. dotted line in Fig. 10(a)]. The slight redshift of the dissociated trion line with respect to the exciton in this electric field range is due to the Coulomb perturbation of the exciton remaining in the right quantum dot by the spectator electron in the left quantum dot. The second electron is removed from the right dot between 60 and $65 \mathrm{kV} / \mathrm{cm}$.

Note, that the observed mechanism of dissociation of the trion into an exciton and an electron does not occur in the system of symmetric quantum dots (cf. Fig. 8). For asymmetric quantum dots the stronger confinement energy in the thinner of the dots prevents the second electron from entering it simultaneously with the first one. In the asymmetric system the exciton becomes dissociated into an electron and a hole for larger electric fields than the one inducing dissociation of the trion into an exciton and a free electron [cf. Fig. 10(b)]. On the other hand the exciton created in the right quantum dot after the trion dissociation is more resistant to the electric field induced dissociation than the exciton. The electron remaining in the right dot is less willing to pass to the left quantum dot if it is already occupied by an electron.

The recombination energy lines of the trion in the asymmetric system of coupled dots present a positive second derivative with respect to the electric field for a certain range of $F$. Namely, for $b=6 \mathrm{~nm}$ the second derivative is positive for the electric field range $F \in(61,71), F \in(57,60)$, and $F \in(50,52) \mathrm{kV} / \mathrm{cm}$ for $b=6,8$, and $10 \mathrm{~nm}$, respectively.

For symmetric dots the mechanism behind the trion dissociation into an electron pair confined in one dot and the hole in the other, without an intermediate step consisting of an exciton in one dot, and the electron occupying the other quantum dot, is easily explained when considering large $b$ using a simple reasoning in which the tunnel effect and the interdot Coulomb interactions are neglected. In this model the dependence of the energy of the trion on the external field can be written as $E_{X^{-}}=-2 E_{e h}+E_{e e}-F b e / 2$, where $E_{e h}$ $\left(E_{e e}\right)$ is the absolute value of the electron-hole interaction for the particles localized in the same dot. The trion is localized in the dot in which the electron localization is favored by the field. The lowest energy level corresponding to the exciton confined in one dot and the electron in the other is $E_{X}$ $=-E_{e h}-F b e / 2$, and the energy level corresponding to a completely dissociated system is $E_{d}=E_{e e}-3 F b e / 2$. For 
$E_{e h}<E_{e e}$ the trion is bound at $F=0$ and at nonzero field the ground state energy equals either $E_{X^{-}}$or $E_{d}$. The energy splitting of $E_{X^{-}}$and $E_{X}$ is not affected by the field which explains the absence of an exciton as an intermediate step of trion ionization. A similar simple reasoning can be used for coupled asymmetric dots where the intermediate step of trion dissociation is now found.

\section{DISCUSSION}

As mentioned in the introduction previous ground-state calculations ${ }^{7,8}$ for the Stark effect in vertically coupled dots detected a deviation of the energy dependence on the electric field from the expected quadratic form obtained within the second order perturbation theory. ${ }^{9}$ The inset to Fig. 1(a) shows that for identical quantum dots this deviation, i.e., a cusp of the recombination energy in function of the electric field, is due to a narrow avoided crossing of two lowest energy levels. In the absence of the electric field these two energy levels are nearly degenerate. This near degeneracy results from the smallness of the hole tunnel coupling between the dots. For the case presented in Fig. 1(a) these two energy levels correspond to opposite parity of the hole [cf. Fig. 2(a) for $F=0$ ]. The electric field easily mixes the two energy levels localizing the hole in the right dot (in the ground state) and in the left quantum dot (in the first excited state). When the confinement potential is asymmetric the discussed anticrossing of the two lowest energy levels are replaced by a crossing (cf. Fig. 6). This is due to a nearly complete localization of the hole in left or right quantum dot in the two states. The cusp of the ground-state is produced by two energy levels crossing or nearly crossing. It is therefore clear that second order perturbation theory for a single nondegenerate energy level given ${ }^{9}$ for a single quantum dot is not applicable to the ground state in coupled quantum dots. There is therefore no reason for which the ground state should follow the quadratic formula and the deviation from parabolicity does not really deserve to be called an anomaly.

In the present paper we have found another deviation from the common quadratic Stark shift, also involving two energy levels. This deviation appears for an intermediate barrier thickness and is due to an avoided crossings of a bright energy level with both carriers in the same dot and a dark energy with separated charge carriers. This unusual Stark effect, shown in Fig. 2(c) for a symmetric dot, should be visible in low-excitation PL spectroscopy. ${ }^{3}$ The observation of the excited exciton states should be facilitated by a relatively weak tunnel coupling between the quantum dots. In the corresponding PL spectrum, one of the lines should be independent of the electric field in both energy and intensity. The additional structure below and above the constantenergy line should be observed in the form of an anticrossing. The intensity of the constant-energy line should be reduced in the region, in which the anticrossing appears.

Real InAs/GaAs quantum dots exhibit a strain-induced intrinsic dipole moment at $F=0 .{ }^{24}$ The intrinsic dipole moment has been neglected in the present calculations. However, the unusual Stark shift for the coupled dots is predicted for quite small electric fields (lower than $15 \mathrm{kV} / \mathrm{cm}$ ), for which the effect of the intrinsic dipole moment is negligible. For comparison in the experiment the intrinsic dipole moment leads to a shift of the transition energy by about $5 \mathrm{meV}$ for $F=100 \mathrm{kV} / \mathrm{cm}^{24}$ Therefore, the intrinsic dipole moment should not modify the qualitative features of the effect predicted in the present paper. The second order effect of the polarizability related to the electric-field induced deformation of the electron and hole wave function for the discussed low electric field range should be even smaller. Similar mechanism of the exciton dissociation via an avoided crossing has been found for asymmetric dots [cf. Figs. 5(a) and 5(b)]. The difference between the ideally symmetric system and the more realistic asymmetric one is that the bright state which does not participate in the avoided crossing is shifted to a different energy, lower or higher depending on the direction of the electric field. The mechanism of the exciton dissociation via an avoided crossing of a dark and a bright energy level described here has been recently confirmed experimentally ${ }^{38}$ after the present paper has been submitted.

Second-order perturbation theory for a single nondegenerate energy level ${ }^{9}$ predicts a nonpositive curvature of the energy level as a function of the electric field. Although the curvature is indeed nonpositive in the ground state, a positive curvature is obtained for the excited bright energy levels in the presence of the avoided crossings with lower energy levels [see Figs. 4(a)-4(c), 9, and 10]. A nondegenerate perturbation theory for a single level ${ }^{9}$ obviously does not apply for the the energy level interaction.

In view of the present results the pronounced drop of the recombination energy for a bias voltage for which an electron is trapped in the quantum $\operatorname{dot}^{5}$ closer to the electron reservoir can be understood provided that the recombination signal in the observed range of wavelengths comes from this dot. Otherwise, the charge of the electron trapped in the dot closer to the reservoir would have a negligible influence on the energy of exciton recombination in the other dot separated by a barrier of $12 \mathrm{~nm}$ [cf. the small energy spacing between the exciton recombination lines with and without a spectator electron in the other dot for $b=10 \mathrm{~nm}$ in Fig. 10(a)]. The drop would result from the electrostatics of the negative trion in which the energy of the electron-hole attraction is larger than the electron-electron repulsion due to a difference of the strength of lateral localization of the carriers (see the discussion given in Ref. 22). The observed growth of the recombination energy for the smaller absolute value of the bias voltage could be related to a passage of one of the electrons to the upper dot. The presented calculations for the trion were limited to the ground state. However, the PL line observed in the experiment which we here attribute to the trion recombination in the lower of the dots does not correspond to the ground state since the quantum dot in the upper layer are larger. Therefore, in the experiment the dissociation of the trion localized in the lower dot could be associated with an avoided crossings with lower energy states, which as obtained for the exciton, can produce a positive curvature of the recombination line over a wide range of electric field values.

\section{SUMMARY AND CONCLUSIONS}

We have studied the exciton and negative trion states in a simple but exactly solvable model of vertically coupled 
quantum dots allowing for a description of the effects related to the modification of the electron-hole interaction by an electric field applied in the growth direction. The effect of the tunnel coupling between the dots and the confinement potential asymmetry was considered. The mechanism of the electric-field induced exciton and trion dissociation was described.

We have shown that the previously ${ }^{7,8}$ found deviations from the quadratic Stark effect are due to energy levels crossings (or very narrow avoided crossings). For weaker tunnel coupling we have found another nonquadratic feature due to an avoided crossing of bright and dark energy levels. This feature appears also in the presence of the asymmetry of the coupled dots and is due to the Coulomb interaction. Positive curvature of the bright excited exciton energy levels is obtained in the range of electric fields corresponding to avoided crossings with lower levels.

Although in the presence of asymmetry of the coupled dots the trion is dissociated into an exciton and an electron by the electric field, for symmetric dots the dissociation mechanism is different, i.e., the trion is directly separated into an electron pair in one dot and the hole in the other. The trion is more stable against this mechanism of dissociation than the exciton. The process of trion dissociation into an exciton and a free electron that we obtain for the case of asymmetric coupling leads to a positive curvature of the PL line as a function of the electric field which has never been observed for the exciton ground state.

\section{ACKNOWLEDGMENTS}

This paper was supported by the Polish Ministry of Scientific Research and Information Technology in the framework of the solicited Grant No. PBZ-MIN-008/P03/2003, the Flemish Science Foundation (FWO-Vl), the Belgian Science Policy, the University of Antwerpen (VIS and GOA) and the European Commission GROWTH programme NANOMAT project, Contract No. G5RD-CT-2001-00545, and the EUNoE SANDiE. T.C. is partly supported by the Marie Curie Training Site Programme of the European Union. B.S. is supported by the Foundation for Polish Science (FNP) and by the EC Marie Curie IEF Project No. MEIF-CT-2004500157. We are thankful for Dr. Ruth Oulton for helpful discussions and for making the experimental data accessible prior to publication.
${ }^{1}$ G. S. Solomon, J. A. Trezza, A. F. Marshall, and J. S. Harris, Phys. Rev. Lett. 76, 952 (1995).

${ }^{2}$ S. Fafard, M. Spanner, J. P. McCaffrey, and Z. Wasilewski, Appl. Phys. Lett. 76, 2707 (2000).

${ }^{3}$ M. Bayer, P. Hawrylak, K. Hinzer, S. Fafard, M. Korkusinski, Z. Wasilewski, O. Stern, and A. Forchel, Science 291, 451 (2001).

${ }^{4}$ B. Szafran, S. Bednarek, and J. Adamowski, Phys. Rev. B 64, 125301 (2001).

${ }^{5}$ R. Oulton, A. I. Tartakovskii, A. Ebbens, J. J. Finley, D. J. Mowbray, M. S. Skolnick, and M. Hopkinson, cond-mat/0407072 (unpublished).

${ }^{6}$ J. H. Ser, Y. H. Lee, J. W. Kim, and J. E. Oh, Jpn. J. Appl. Phys., Part 1 39, 518 (2000).

${ }^{7}$ W. Sheng and J.-P. Leburton, Phys. Rev. Lett. 88, 167401 (2002).

${ }^{8}$ K. L. Janssens, B. Partoens, and F. M. Peeters, Phys. Rev. B 65, 233301 (2002).

${ }^{9}$ J. A. Barker and E. P. O'Reilly, Phys. Rev. B 61, 13840 (2000).

${ }^{10}$ M. Grundmann, O. Stier, and D. Bimberg, Phys. Rev. B 52, 11969 (1995).

${ }^{11}$ M. A. Cusack, P. R. Briddon, and M. Jaros, Phys. Rev. B 54, R2300 (1996).

${ }^{12}$ C. Pryor, Phys. Rev. B 57, 7190 (1998); 60, 2869 (1999).

${ }^{13}$ O. Stier, M. Grundmann, and D. Bimberg, Phys. Rev. B 59, 5688 (1999).

${ }^{14}$ L. Landin, M. E. Pistol, C. Pryor, M. Persson, L. Samuelson, and M. Miller, Phys. Rev. B 60, 16640 (1999).

${ }^{15}$ R. Heitz, O. Stier, I. Mukhametzhanov, A. Madhukar, and D. Bimberg, Phys. Rev. B 62, 11017 (2000).

${ }^{16}$ A. J. Williamson and A. Zunger, Phys. Rev. B 61, 1978 (2000).

${ }^{17}$ W. Sheng and J.-P. Leburton, Phys. Rev. B 63, 161301(R) (2001).

${ }^{18}$ F. Guffarth, R. Heitz, A. Schliwa, O. Stier, N. N. Ledentsov, A. R. Kovsh, V. M. Ustinov, and D. Bimberg, Phys. Rev. B 64,
085305 (2001).

${ }^{19}$ F. Guffarth, R. Heitz, A. Schliwa, O. Stier, M. Geller, C. M. A. Kapteyn, R. Sellin, and D. Bimberg, Phys. Rev. B 67, 235304 (2003).

${ }^{20}$ B. Urbaszek, R. J. Warburton, K. Karrai, B. D. Gerardot, P. M. Petroff, and J. M. Garcia, Phys. Rev. Lett. 90, 247403 (2003).

${ }^{21}$ B. Stébé and A. Ainane, Superlattices Microstruct. 5, 545 (1989); C. Riva, F. M. Peeters, and K. Varga, Phys. Rev. B 61, 13873 (2000).

${ }^{22}$ B. Szafran, B. Stébé, J. Adamowski, and S. Bednarek, Phys. Rev. B 66, 165331 (2002).

${ }^{23}$ E. Anisimovas and F. M. Peeters, Phys. Rev. B 68, 115310 (2003).

${ }^{24}$ P. W. Fry, I. E. Itskevich, D. J. Mowbray, M. S. Skolnick, J. J. Finley, J. A. Barker, E. P. O'Reilly, L. R. Wilson, I. A. Larkin, P. A. Maksym, M. Hopkinson, M. Al-Khafaji, J. P. R. David, A. G. Cullis, G. Hill, and J. C. Clark, Phys. Rev. Lett. 84, 733 (2000).

${ }^{25}$ F. Troiani, U. Hohenester, and E. Molinari, Phys. Rev. B 65, 161301(R) (2002).

${ }^{26}$ V. Halonen, T. Chakraborty, and P. Pietiläinen, Phys. Rev. B 45, 5980 (1992).

${ }^{27}$ K. T. Davies, H. Flocard, S. Kreger, and M. S. Weiss, Nucl. Phys. A 342, 112 (1980).

${ }^{28}$ F. M. Peeters and J. E. Golub, Phys. Rev. B 43, 5159 (1991).

${ }^{29}$ A. Wójs, P. Hawrylak, S. Fafard, and L. Jacak, Phys. Rev. B 54, 5604 (1996).

${ }^{30}$ B. Szafran, B. Stébé, J. Adamowski, and S. Bednarej, J. Phys.: Condens. Matter 12, 2453 (2000).

${ }^{31}$ S. Glasberg, G. Finkelstein, H. Shtrikman, and I. Bar-Joseph, Phys. Rev. B 59, R10 425 (1999).

${ }^{32}$ A. V. Filinov, C. Riva, F. M. Peeters, Yu. E. Lozovik, and M. 
Bonitz, Phys. Rev. B 70, 035323 (2004).

${ }^{33}$ B. Stébé and A. Moradi, Phys. Rev. B 61, 2888 (2000).

${ }^{34}$ A. Esser, R. Zimmermann, and E. Runge, Phys. Status Solidi B 227, 317 (2001).

${ }^{35}$ S. Bednarek, B. Szafran, T. Chwiej, and J. Adamowski, Phys. Rev. B 68, 045328 (2003).

${ }^{36}$ For the trion, unlike for the exciton, the value of the dipole moment depends on the choice of the origin. The values displayed in the inset of Fig. 8(b) are calculated for the origin set in the middle of the barrier.
${ }^{37}$ If the ground state of a single electron was localized in the left dot for higher value of the electric field, the recombination energy, calculated as the difference of the ground state of the trion and electron, would have a doubtful physical interpretation since it would additionally assume that the recombination of the electron-hole pair is accompanied by the passage of the electron from the right to the left dot.

${ }^{38}$ H. J. Krenner, M. Sabathil, E. C. Clark, A. F. Kress, D. Schuh, M. Bichler, G. Abstreiter, and J. J. Finley, Phys. Rev. Lett. 94, 057402 (2005). 